\title{
Pathophysiology and management of alzheimer's disease: an overview
}

\begin{abstract}
Alzheimer's disease (AD) is the sixth-leading reason of fatality and is $70 \%$ present in all cases of dementia. The global burden of AD is expected to accelerate from 26.6 million cases in 2006 to 106.8 million by 2050 . The total assumed worldwide costs of dementia were US $\$ 604$ billion in 2010, equivalent to $1 \%$ of the world's Gross domestic product (GDP). This review is focused on the overview of pathophysiology and management of AD. The sources for the literature search were PubMed, ScienceDirect, Scopus and Google Scholar. AD is generally considered as disorders related to intensify loss of neurons and synapses proceeds in distinct anatomical loci, resulting in different phenotypes. The pathophysiology of Alzheimer's disease is credited to a number of factors such as the cholinergic dysfunction, amyloid/tau toxicity and oxidative stress/ mitochondrial dysfunction. Apart from various therapeutic targets, biomarkers and pharmaco-therapies available; Herbal drugs, secondary metabolites and to the some extent non-pharmacological therapies are impending and potential candidates for the management of AD. Further studies are required to elaborate the detailed mechanism of Herbal drugs, secondary metabolites and non-pharmacological therapies for the management of $\mathrm{AD}$.
\end{abstract}

Keywords: alzheimer's disease, amyloid- $\beta$ peptide, mitochondrial dysfunction, biomarkers, herbal drugs
Volume 7 Issue 2 - 2018

\author{
Ajit Kumar Thakur,' Parul Kamboj,' Kritika \\ Goswami,' Karan Ahuja² \\ 'School of Pharmaceutical Sciences, Delhi Pharmaceutical \\ Sciences and Research University, India \\ ${ }^{2}$ Delhi Institute of Pharmaceutical Sciences and Research, Pushp \\ Vihar Sector-III, India
}

\begin{abstract}
Correspondence: Ajit Kumar Thakur, Assistant Professor of Pharmacology, School of Pharmaceutical Sciences, Delhi Pharmaceutical Sciences and Research University, Pushp Vihar Sector-III, M.B. Road, New Delhi- I 0 0 I7, India, Fax 9|-I I29554503,Email ajitmitpharm@gmail.com
\end{abstract}

Received: February 28, 2018| Published: April 26, 2018

\section{Introduction}

Alzheimer's disease (AD) concedes as progressive neurodegenerative disorder, the foremost cause of dementia in late adult life. Intracellular neurofibrillary tangles (NFTs) and extracellular amyloidal protein deposits as the senile plaques characterize it pathologically. Accumulations of $A \beta$ are amyloid plaques in the brain parenchyma and in the cerebral blood vessels where it is known as congophilic angiopathy also known as cerebral amyloid angiopathy (CAA). NFTs formed the paired helical filaments with hyperphosphorylated tau proteins. These NFTs characterized by the neuronal and synaptic loss and some certain distinctive lesions. Various treatments are available for the disease which include acetylcholinesterase inhibitors (rivastigmine, galantamine, donepezil) and $\mathrm{N}$-methyl D-aspartate receptor antagonist (memantine). ${ }^{1}$ Traditionally, Alzheimer's disease considered as disorders in which intensifying loss of neurons and synapses proceeds in distinct anatomical loci, resulting in different phenotypes. The diagnosis of $\mathrm{AD}$ is done by evaluating the location, distribution, and abundance of characteristic brain lesions. These neuroimaging techniques are emerging and capable of both quantifying AD-associated cerebral atrophy and detecting amyloid $\beta(\mathrm{A} \beta$ ) peptide, phosphorylated tau (phospho-tau), or other $\beta$-pleated sheet proteins in the brain while patients are alive, these techniques also help when disease is completely asymptomatic or at early stages of neurodegeneration. Autopsy or biopsy neuropathologic is to be considered the gold standard for the diagnosis of $\mathrm{AD}$ and non-AD dementias. The important characteristics feature of the $\mathrm{AD}$ brain on is cortical atrophy, commonly it diffuse and fairly commensurate throughout the cerebral hemispheres rather than being prominent in certain lobes or on one side of the brain (as in the case of some fronto-temporal lobar degenerations). Fresh brain weight is usually below the normal range for an adult $(1,200-1,400 \mathrm{~g})$, though not necessarily so it may be entirely normal and even, rarely, above the upper range of normal. When the fixed brain is sliced, the cortical atrophy (manifest as thinning of the cortical ribbon) is usually accompanied by enlargement of the ventricular system, or hydrocephalus ex-vacuo, and sometimes by shrinkage, atrophy, and/ or pallor of the subcortical white matter. Coronal slices of (fixed) brain from two different patients, one without dementia and one with; the slices are at comparable coronal levels (near the head of the caudate nucleus). Cortical thinning is less prominent. ${ }^{2}$ Alzheimer's disease is characterized by a gradual decrease in the cognitive function, which includes a decline in memory, learning capacity of individuals and also thinking capacity and it is significantly increased among people of age 65 years or above. The disease often begins with minor symptoms and ends with severe brain damage or even death. $1 \%-6 \%$ is the premature stage of all Alzheimer's disease (age $<60-65$ years) and approximately $60 \%$ of premature-stage $\mathrm{AD}$ is familial, with $13 \%$ appearing to be inherited in an autosomal prevailing manner. The difference between premature-stage familial Alzheimer disease (onset age $<60-65$ years) and late stage familial Alzheimer disease (onset age $>60-65$ years) is somewhat erratic. ${ }^{3}$ Demise of an individual suffering from AD usually results from general starvation, malnutrition, and pneumonia. ${ }^{4}$ The substantial growth of the disease can be categorized in four different stages. The first stage is the Mild Cognitive Impairment (MCI), and shows a range of symptoms (usually amnesia) which does not alter daily life significantly. Between 6 and $25 \%$ of people affected with MCI develop AD every year. The later stages of Alzheimer's disease (mild and moderate AD) are characterized by an augment in cognitive deficits, and a decline in independence, culminating in the patient's complete dependence on caregivers and a complete deterioration of personality (Severe AD). Alzheimer's disease is the sixth-leading 
reason of fatality and is $70 \%$ present in all cases of dementia. Other symptoms of Alzheimer Disease include perplexity, improper judgment, irritation, withdrawal, and hallucinations. Sometimes, seizures, Parkinsonian features, amplified muscle tone, myoclonus, incontinence, and mutism may occur. ${ }^{3}$ Primary care of patients should be taken for recognition and supervision of disease, where they may be presented with various diagnostic and management challenges. This helps in early investigation and diagnosis of Alzheimer's disease and ultimately it leads to symptomatic pharmacological treatments and psychosocial support. ${ }^{5}$

\section{Global economic of AD}

The global burden of $\mathrm{AD}$ is expected to accelerate from 26.6 million cases in 2006 to 106.8 million by $2050 .{ }^{3}$ The total assumed worldwide costs of dementia were US\$ 604 billion in 2010, equivalent to $1 \%$ of the world's GDP. Low-income countries accounted for just less than $1 \%$ of total worldwide costs (but $14 \%$ of the prevalence of dementia), middle-income countries for $10 \%$ of the costs (but $40 \%$ of the prevalence of dementia) and high-income countries for $89 \%$ of the costs (but $46 \%$ of the prevalence of dementia). About $70 \%$ of the global costs occurred in just two regions: Western Europe and North America. These discrepancies are accounted for by the much lower cost per person in lower income countries - US\$ 868 in low-income countries, US\$ 3,109 in lower-middle-income, US\$ 6,827 in uppermiddle-income, and US\$ 32,865 in high-income countries. ${ }^{6}$ In highincome countries, informal care $(45 \%)$ and formal social care $(40 \%)$ account for the major costs, while the proportionate contribution of direct medical costs $(15 \%)$ is much lower. In low-income and lower-middle-income countries, direct social care costs are minute and informal care costs (i.e. unpaid care provided by the family) predominate. Changing population demographics in many LMIC may lead to a decline in the ready availability of extended family members in the coming decades" states the 2012 WHO report "Dementia: a health priority.

\section{Pathophysiology of AD}

Neuronal loss and/or pathology may be seen particularly in the hippocampus, amygdala, entorhinal cortex and the cortical association areas of the frontal, temporal and parietal cortices, but also with subcortical nuclei such as the serotonergic dorsal raphe, noradrenergic locus coeruleus, and the cholinergic basal nucleus. The deposition of tangles follows a defined pattern, starting from the trans-entorhinal cortex; consequently the entorhinal cortex, the CA1 region of the hippocampus and then the cortical association areas, where frontal, parietal and temporal lobes are particularly affected. The extent and placement of tangle formation correlates well with the severity of dementia, much more so than numbers of amyloid plaques.

The accretion of tau proteins correlates very closely with cognitive decline and brain atrophy, including hippocampal atrophy. In the neuropathology of Alzheimer's disease there is a loss of neurons and atrophy in temporofrontal cortex, which causes inflammation and deposit the amyloid plaques and an abnormal cluster of protein fragments and tangled bundles of fibers due to this there is an increase in the presence of monocytes and macrophages in cerebral cortex and it also activates the microglial cells in the parenchyma. ${ }^{7}$ Summary of pathophysiology of AD are shown in Figure 1.

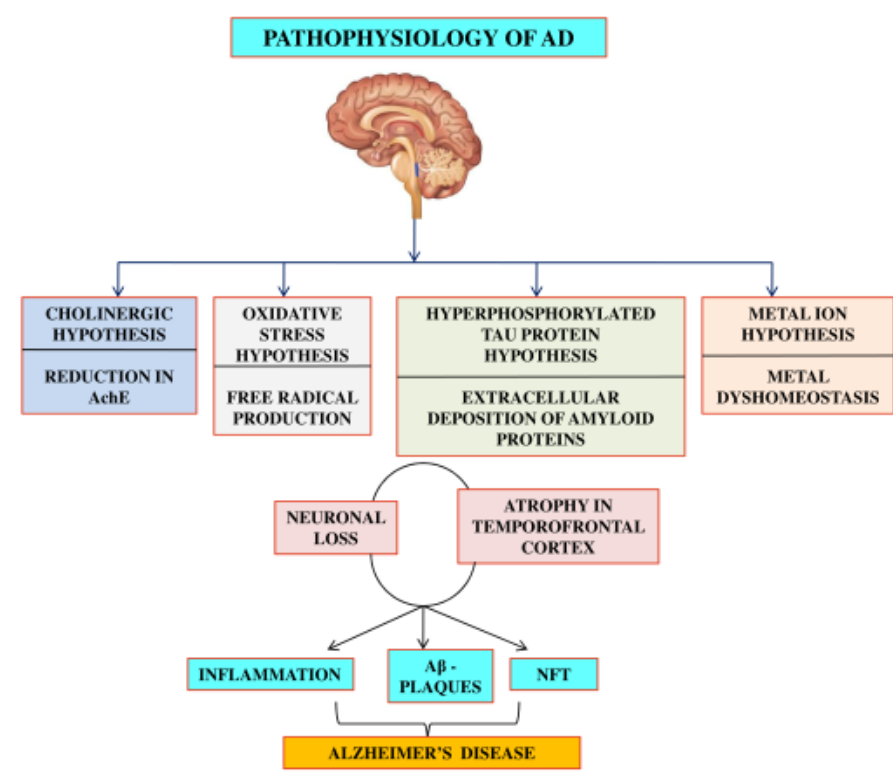

Figure I Hypothesis for pathophysiology of Alzheimer's disease.

\section{Hyperphosphorylated tau protein and amyloid $\beta$ hypothesis}

One of the main pathological features of $\mathrm{AD}$ is the formation of senile plaques (SP), which is caused by amyloid beta $(A \beta)$ deposition. Normally, $A \beta$ are soluble small peptides, which are produced by the splitting of the precursor protein of amyloid (APP) by the action of $\alpha$-secretase, $\beta$-secretase and $\gamma$-secretase. The imbalance between $\beta$-amyloid $(\mathrm{A} \beta)$ production and clearance leads to various types of toxic oligomeric, namely protofibrils, fibrils and plaques depending upon the extent of oligomerization. The reason of the formation of $A \beta$ is still unclear, but the sequence, concentration and conditions of stability of $A \beta$ are important factors. ${ }^{8}$ The pathophysiology of Alzheimer's disease is credited to a number of factors such as the cholinergic dysfunction, amyloid/tau toxicity and oxidative stress/ mitochondrial dysfunctions. ${ }^{9}$

\section{Oxidative stress hypothesis}

Reactive oxygen species (ROS) and reactive nitrogen species (RNS) are produced in many normal and abnormal processes in humans, they play dual role as both have beneficial functions in cellular signaling pathways and venomous processes that can lead to damage of cellular structures (including cell membrane, lipid, protein, and DNA). The high oxygen consumption of the brain, which utilizes $20 \%$ more oxygen than other mitochondrial respiratory tissues, means that the brain is more vulnerable to oxidative stress. The neuron is the basic functional unit of the brain, which contains a large number of polyunsaturated fatty acids. It can interact with ROS, leading to the lipid peroxidation reaction and molecular apoptosis, in addition, less glutathione in neurons is also one of the causes of oxidative stress injury. ${ }^{8}$

\section{Metal ion hypothesis}

Metal dyshomeostasis is involved in the progression and pathogenesis of diseases, including neurodegerative diseases and 
cancer. Ionosphere and metal chelators are well known modulators of transition metal homeostasis, and a number of these molecules are used in clinical trials. Metal-binding compounds are not the only drugs capable of targeting transition metal homeostasis. ${ }^{10}$ Current evidence indicates changes in the equilibrium of redox transition metals; mainly copper $(\mathrm{Cu})$, iron $(\mathrm{Fe})$ and other trace metals. Their levels in the brain are found to be high in $\mathrm{AD}$. In other neurodegenerative disorders, $\mathrm{Cu}$, manganese, aluminum and zinc are involved. ${ }^{11}$

\section{Cholinergic hypothesis}

The effects of apo-lipo-protein E (APOE) genotype on the useful effect of acetyl-cholinesterase inhibitors (AChEIs) in patients with Alzheimer's disease. AchEI medications are the core of the treatment of $\mathrm{AD}$, and APOE genotype is the most important factor associated with AD. This lack of major effect of APOE is analyzed with respect to the "Cholinergic Hypothesis" of AD, dating from 1976, through the recognition that cholinergic neurons are not the main target of $\mathrm{AD}$.

Cholinergic receptor binding is reduced in specific brain regions with mild to moderate $\mathrm{AD}$ and is related to neuropsychiatric symptoms. Among healthy older adults, lower receptor binding may be associated with slower processing speed. Cholinergic receptor binding in vivo may reveal links to other key brain changes associated with aging and $\mathrm{AD}$ and may provide a potential molecular treatment target. ${ }^{12}$ Clinical decrease is related to an extensive loss of cholinergic neurons formed in the forebrain nuclei (medial) and a related decline in acetylcholine-mediated neurotransmission, drugs tending to regularize acetylcholine transmitter level, such as cholinesterase inhibitors (ChEIs) and donepezil, have for over 20 years served as the foundation of symptomatic therapy for $\mathrm{AD} .^{13}$

\section{Clinical criteria}

The diagnostic criteria of Alzheimer's disease is based on the National Institute of Neurological and Communicative Disorders and Stroke (NINCDS) and the Alzheimer's Disease and Related Disorders Association (ADRDA) criteria, according to this the diagnosis is categorized as definite (clinical diagnosis with histological confirmation), probable (typical clinical syndrome without histological confirmation), or possible (atypical clinical features but no alternative diagnosis apparent; no histological confirmation. ${ }^{14}$ The recent guiding principle elaborates the concept of Alzheimer's dementia beyond memory loss as its primary symptom and incorporates the possibility that a decrease in other aspects of cognition, such as anomia and impaired executive functioning may be the primary symptoms to be identified. Biomarkers may be used to increase the specificity of diagnosis. ${ }^{15}$ Clinicians used the term $\mathrm{AD}$ to refer to a clinical dementia entity that basically presents with a specific progressive amnesic disorder following the appearance of other cognitive and neuropsychiatric changes that impair social function and activities of daily living. In the NINCDS-ADRDA criteria, biological investigation (blood and cerebrospinal fluid [CSF]) and neuro imaging examination (computed tomography [CT] scan or magnetic resonance [MR] imaging) were only proposed to exclude other causes of the dementia syndrome (example- vascular lesions, tumors, infectious or inflammatory processes). Typical sensitivity and specificity values for the diagnosis of probable $\mathrm{AD}$ with the use of NINCDS-ADRDA criteria are $81 \%$ and $73 \%$ respectively. ${ }^{14}$ Many therapeutic approaches are used to get better the cholinergic neurotransmission, but their function in the pathogenesis of $\mathrm{AD}$ is still mystified. Although, enhance in tau protein focus in CSF is described in $\mathrm{AD}$, but many issues remain ambiguous. Accurate and extensive analysis of CSF could be useful to describe the existence of tau proteins in physiological conditions, or released during the development of neurodegenerative disease. The amyloid cascade theory postulates that the neurodegeneration in $\mathrm{AD}$ caused by the abnormal accretion of amyloid beta (A $\beta)$ plaques in many areas of the brain. ${ }^{16}$ Plasma concentrations of free $A \beta$ could not predict the development of clinical $\mathrm{AD}$, and $\mathrm{A} \beta$ concentrations did not change in the years preceding $\mathrm{AD}$ diagnosis. ${ }^{17}$

The clinical diagnosis of Alzheimer's disease is described as a sequence: first step includes the history of the patient and most important information of patient about previous diseases from an informant. The second step includes the evaluation of mental state and confirmatory cognitive function test. The third and important step includes the physical examination which should focus on vascular and neurological signs supplemented by investigations. Assessment of dementia is a two step process. Firstly, it is important differentiation of dementia syndromes from other conditions that minimize them, these conditions are depression, delirium, and mild cognitive impairment. Secondly, once the recognition of dementia syndrome is done, the diagnosis of dementia subtype is important because it helps in choosing the possible treatment of disease. ${ }^{5}$ Early and accurate diagnosis of Alzheimer's disease has a key impact on the progress of research on dementia. To address the problems involved in diagnosing $\mathrm{AD}$ in its earliest stages, the National Institute on Aging, the American Association of Retired Persons, the National Institute of Neurological and Communicative Disorders and Stroke, and the National Institute of Mental Health jointly sponsored a workshop for planning research. ${ }^{18}$ Essentials of the diagnostic work up of Alzheimer includes clinical exam, medical history of the patient, assessment of multiple cognitive domains, lab tests, MRI and/or CT scan, in some cases. Clinical examination is mostly marked by the loss of the ability to encode information, with additional signs of aphasia and anoxia, but neurologic reflexes are otherwise normal. As symptoms progresses, patients may show bewilderment, psychosis, screaming, and fluctuation as symptoms. In the terminal state, after 4.5 years of illness, the patient was bed ridden, contracted, and incontinent. ${ }^{18}$ The Diagnosis of Alzheimer's disease can be done on the basis of clinical criteria which include precarious onset and gradual destruction of cognitive functions and memory. In the early stage of disease, there are no sensory and motor dysfunction and coordination deficits. Determination of the disease cannot be done by laboratory tests, although these tests are important mainly in the identification of other probable causes of dementia and these causes should be disqualified before the diagnosis of disease with surety. Neuropsychological tests for diagnosis of dementia and these test help to evaluate the result of therapy do confirmation of the disease.

The diagnosis of "definite Alzheimer's disease" requires a postmortem brain examination and the demonstration of numerous senile plaques and neurofibrillary tangles in hippocampal and association cortical areas. The neuropathological examination confirms probable Alzheimer's disease clinical diagnosis in $85 \%$ of the cases examined in medical schools. However, with much more than $15 \%$ errors, the early diagnosis of Alzheimer's disease must be improved since it is a main factor in the therapeutic approach, and more especially for the efficiency of drug trials. Now, there are new leads for a biological diagnosis in the blood or the CSF. However, the natural (and molecular) history of Alzheimer's disease points out that all biochemical dysfunctions remain in the CNS, and most particularly in association 
brain areas. This is demonstrated using reliable biochemical markers such as Amyloid beta and pathological Tau proteins, which are the basic components of amyloid deposits and neurofibrillary tangles, respectively. Also, a genetic diagnosis can be performed in half of familial autosomic dominant Alzheimer's disease, which represents less than $1 \%$ of all Alzheimer's disease cases. ${ }^{19}$ Doctors to diagnose the patient's condition and the stage as early as possible commonly use Alzheimer's screening tests. Most of these tests are based on penpaper interaction. ${ }^{19}$ Current diagnostic standards NINCDS-ADRDA and the DSM-IV-TR are the criteria for Alzheimer's disease in research; however, they are not matched with the growth of scientific knowledge. Through structural MRI, molecular neuroimaging with PET, and cerebrospinal fluid analysis technique distinct and consistent biomarkers of $\mathrm{AD}$ are now available through structural MRI, molecular neuroimaging with PET, and cerebrospinal fluid analyses. ${ }^{20}$ It is highly prudent to discover reliable biomarkers, to detect its exact pathophysiology during pre-symptomatic stage. High sensitivity and specificity Biomarker(s) would make AD diagnosis possible at early stage. CSF amyloid $\beta 1-42$, total tau, and phosphorylated tau181 presently used as $\mathrm{AD}$ biomarkers. ${ }^{21}$ It is highly prudent to discover reliable biomarkers, to detect its exact pathophysiology during presymptomatic stage. High sensitivity and specificity Biomarker(s) would make AD diagnosis possible at early stage, CSF amyloid $\beta 1-42$, total tau, and phosphorylated tau181 presently used as AD biomarkers. ${ }^{21}$

\section{Biomarkers for AD}

Biomarkers can also be of great value in Alzheimer's disease drug development to select the most optimal drug candidates for large and expensive phase 3 clinical trials. Biomarkers will also be important to provide evidence that a drug affects the underlying pathophysiology of the disease, which, together with a beneficial effect on the clinical course, will be essential for labeling the drug as having a diseasemodifying effect. ${ }^{22}$ Summary of various biomarkers of AD are depicted in Figure 2A.

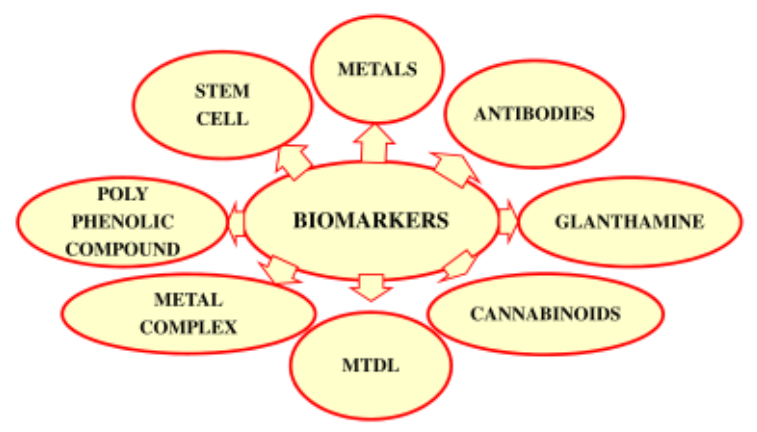

(A)

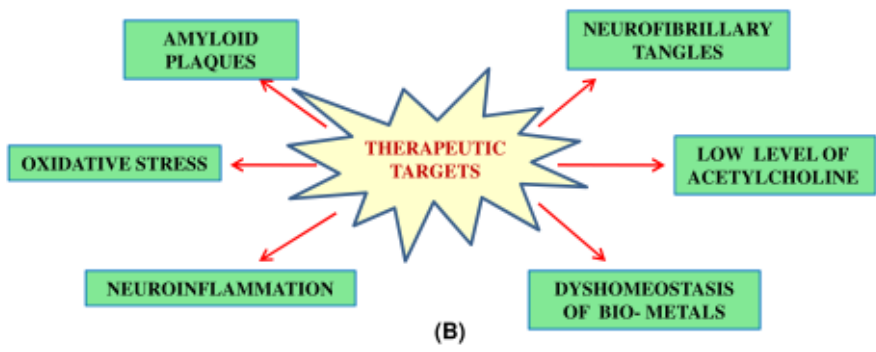

Figure 2 Biomarkers (A) and therapeutic targets (B) for management of Alzheimer's disease.

\section{Galanthamine}

In current years, isolation of galanthamine is done by numerous members of the Amaryllidaceae (Leucojum spp., Narcissus species, Galanthus spp.) has turned out to be an essential therapeutic options used to delay the process of degeneration of neurons in Alzheimer's disease. ${ }^{23}$ Galanthamine is well tolerated during long-term treatment. Galanthamine under the name of Nivalin is already approved in Austria for $\mathrm{AD}$ and is marketed there and in Germany for other indications such as facial neuralgia. ${ }^{24}$

\section{Cannabinoids}

Cannabinoid system has become a potential therapeutic approach to treat $\mathrm{AD}$. The cannabinoid is composed of a number of cannabinoid receptors $(\mathrm{CB})$ including well characterized $\mathrm{CB} 1$ and $\mathrm{CB} 2$ receptors. Findings indicate that the activation of both $\mathrm{CB} 1$ and $\mathrm{CB} 2$ receptors by natural or synthetic agonists at non psychoactive doses have beneficial effects in Alzheimer experimental models by reducing harmful beta amyloid peptide action and tau phosphorylation, as well by promoting brain's intrinsic repair mechanisms. ${ }^{25}$

\section{Antibodies}

Intravenous immunoglobulin or purified $A \beta$ antibodies may be suggested as a possible treatment as it modifies $A \beta$ and $A \beta 1$ - levels, suggesting potential utility as a therapy for Alzheimer's disease. ${ }^{26}$

\section{MTDL}

Multi-target-directed ligand (MTDL) moves toward with the promising method in the multifactorial aetiology of the disease to search for new drugs of AD. The most of the novel molecules having heterodimeric structures they are capable of interacting with no. of targets in combination with pharmacophores, they may be of natural origin and original or preexisting drugs (tacrine, donepezil, galantamine, memantine). Some drug which is described here appears to be promising drugtreatment, while others may act as a helpful inspiration to search new and effective therapies for $\mathrm{AD} .{ }^{27}$

\section{Metal complexes}

Luminescent ruthenium and iridium metal complexes with long fluorescent lifetimes that undergo significant changes to their electronic spectra upon binding to $A \beta$ fibrils have potential as useful probes to gain increased insight into the molecular aspects of amyloid formation and aggregation. Complexes with the kinetically inert ions interact with $A \beta$ peptide of varying length to give adducts that have altered toxicity and propensity to aggregate. The viability of metal complexes to be therapeutically useful inhibitors of amyloid formation and $A \beta$ toxicity will be dependent on them satisfying to stringent and challenging requirements of therapeutic agents. ${ }^{28}$

\section{Poly phenolic compounds}

Polyphenolic compounds have been increasingly viewed as natural sources for treating numerous illnesses including, neurological disorders due to their strong antioxidant, anti inflammatory, anti microbial, and antitumor activities. Mounting evidences suggest that polyphenolic compounds, from a variety of diverse sources are able to improve cognitive function and reduce brain neuropathy in animal models of AD through multiple mechanisms. ${ }^{29}$

\section{Metals}

Lithium has also been regarded as a neuroprotective agent and a 
candidate drug for disease-modification in certain neurodegenerative disorders, namely, Alzheimer's disease. The putative neuroprotective effects of lithium rely on the fact that it modulates several homeostatic mechanisms involved in neurotrophic response, autophagy, oxidative stress, inflammation, and mitochondrial function. Such a wide range of intracellular responses may be secondary to two key effects, that is, the inhibition of glycogen synthase kinase-3 beta (GSK-3 $\beta$ ) and inositol monophosphatase (IMP) by lithium. ${ }^{30}$

\section{Stem cells}

Recent preclinical evidence suggests that stem cells can be used in the treatment of AD. The processes of stem cell based therapies of AD include stem cell mediated neuroprotection and trophic action, anti amyloidogenesis, beneficial modulation and replacement of neurons. $\mathrm{AD}$ can be modeled by iPSC, investigate familial and sporadic AD pathogenesis and screen for anti-AD drugs. ${ }^{30}$

\section{Therapeutic targets for alzheimer disease}

Pathologically Alzheimer's disease is characterized by intracellular neurofibrillary tangles and extracellular amyloid protein deposits contributing to senile plaques. ${ }^{1}$ The study of diseaseinitiating mechanisms and AD progression in humans is inherently difficult as most available tissue specimens are from late-stages of the disease. Therefore, $\mathrm{AD}$ researchers rely on in vitro studies and the use of $\mathrm{AD}$ animal models where neuroinflammation was a major characteristic of AD. Purinergic receptors are a diverse family of proteins consisting of $\mathrm{P} 1$ adenosine receptors and $\mathrm{P} 2$ nucleotide receptors for ATP, UTP and their metabolites. This family of receptors has been shown to regulate a wide range of physiological and pathophysiological processes, including neuroinflammation, and may contribute to the pathogenesis of neurodegenerative diseases like AD. ${ }^{31}$ Different hallmarks of the disease have been reported such as deposits of $\beta$-amyloid around neurons, hyperphosphorylated tau protein, oxidative stress, dyshomeostasis of bio-metals, low levels of acetylcholine, etc. ${ }^{32}$ Various therapeutic targets for AD are summarized in Figure 2B. G-protein coupled receptors are the most abundant receptor type in the central nervous system and are linked to complex downstream pathways, the manipulation of which may have therapeutic applications. ${ }^{33}$ Amyloid $\beta$ hypothesis places the $A \beta$ peptide as the principal factor that triggers the pathological cascade that leads to the dementia and other symptoms of AD. ${ }^{34}$ Studies have shown that mitochondrial dysfunction plays an important role in $\mathrm{AD}$, in which oxidative stress-induced respiratory chain dysfunction, loss of mitochondrial biogenesis, defects of mitochondrial dynamics and mtDNA mutations are observed. Drug therapy target for AD might be mitochondria. Current treatments for AD are not directly targeted to mitochondria. Cholinesterase inhibitors (galantamine, donepezil and rivastigmine) and N-methyl-d-aspartate receptor antagonist memantine are the only two kinds of drugs approved by the FDA for $\mathrm{AD}$ treatment. ${ }^{35}$

There is no cure for $\mathrm{AD}$, and drug therapy for the disease is still in its infancy. Medications, which are approved for the treatment of probable $\mathrm{AD}$ help, have power over the symptoms of $\mathrm{AD}$, but do not slow down the progression or reverse the course of the disease itself. The comprehensive management of $\mathrm{AD}$ entails both nonpharmacological and pharmacologic interventions. Non-pharmacological interventions primarily address behavioral disturbances (e.g. task simplification, environmental modification, minimal excess stimulation, etc.) and other sources of cognitive impairment (e.g., treating comorbid medical conditions, minimizing or eliminating drugs with deleterious cognitive side effects). Pharmacologic strategies have focused on modulating disease-associated neurotransmitter alterations; strategies can be characterized as symptomatic or neuroprotective. Although a symptomatic and a neuroprotective pharmacologic treatment may have similar outcome characteristics in a clinical trial, the key difference is that a neuroprotective therapy will have a cumulative benefit that persists after the treatment is discontinued. Currently available treatments, including cholinesterase inhibitors (ChEIs) and N-methyl-D-aspartate (NMDA) receptor antagonists, are measured symptomatic treatments based on their capacity to slow the clinical development of symptoms across cognitive, behavioral, and functional domains. Initial pharmacologic strategies for $\mathrm{AD}$ focused on increasing cholinergic transmission in the brain based on the "cholinergic hypothesis" of memory dysfunction. Among the different strategies employed to increase synaptic levels of acetylcholine (ACh), blocking the breakdown of ACh by inhibiting acetylcholinesterase (AChE) has proven most successful to date. Inhibiting the enzyme butyrylcholinesterase (BuChE), which is a minor constituent in normal brains, but in the brains of $\mathrm{AD}$ patients is increased in association with plaques and tangles, may also improve cholinergic transmission. ${ }^{36}$

Current pharmacological treatments of $\mathrm{AD}$ reduce symptoms without affecting the main pathological characteristics of the disease. Therefore, it is essential to develop effective and new therapies. There are various potential treatments of Alzheimer's disease. New therapeutic approaches, including those more closely targeted to the pathogenesis of the disease are under research. These potentially disease modifying treatments include amyloid- $\beta$-peptide vaccination, secretase inhibitors, cholesterol lowering drugs, metal chelators and anti-inflammatory agents. ${ }^{37}$ A number of potential therapies are currently under investigation, including estrogen replacement, anti-inflammatory agents, free radical scavangers and antioxidants, and monoamine oxidase-B (MAO-B) inhibitors. The evidence for a protective effect of estrogen or non-steroidal anti-inflammatory drugs (NSAIDs) is controversial and largely based on retrospective studies. More controlled prospective studies are needed to definitively demonstrate the benefits of long term estrogen or NSAID use in prevention of $\mathrm{AD}$. Free radicals scavangers or antioxidants such as idebenone, and selective prevention MAO-B inhibitors such as Lazabemide are well tolerated, but require additional studies in order to demonstrate preventive effects. In addition, their approaches, such as anti-amyloid treatments that affect beta amylase secretion, aggeregation and toxicity, appear promising; treatments that hinder neurofibrillary tangle construction and nerve growth factor (NGF) induction are in the very early stages of development. ${ }^{38} \mathrm{~A}$ considerable effort has been spent investigating the therapeutic potential of antioxidants and anti-inflammatory agents, several of natural products and dietary origin, in $\mathrm{AD}$ treatment. Numerous drug targets have also been investigated for $\mathrm{AD}$ treatment and a modest drug pipeline is available. Despite these efforts, drug development for AD has proved extremely difficult and most clinical trials have afforded disappointing results. ${ }^{39}$ Anti inflammatory drugs are a potential target which, when built upon could be an effective treatment of Alzheimer's disease. The association of immune proteins and immune-competent microglial cells with senile plaques (SP) in both $\mathrm{AD}$ and normal aging suggests that these drugs may be able to modify the course of Alzheimer's disease, either by interfering with Senile Plaques 
formation or by suppressing the inflammation associated with the Senile Plaques. ${ }^{39}$ Non genetic changes, including DNA methylation and histone modifications are involved in learning and memory and have been recently highlighted for holding promise as potential targets for AD therapeutics. Dynamic and latent epigenetic alterations are incorporated in $\mathrm{AD}$ pathological pathways and present valuable reversible targets for $\mathrm{AD}$ and other neurological disorders. ${ }^{40}$ Alteration in brain insulin metabolism has been suggested as one pathological factor underlying this neurodegenerative disorder. In continuing with this theory, patients with Alzheimer's disease show decreased brain insulin receptor sensitivity. Insulin mitigates the hippocampal synapse vulnerability to beta amyloid, a peptide thought to be causing the development of Alzheimer's disease, provide a strong rationale for hypothesizing that pharmacological strategies strengthening brain insulin signaling, such administration of insulin(intra nasaly), could have significant potential in the treatment and prevention of AD. ${ }^{41}$

\section{Pharmacotherapeutics of AD}

Only four drugs are currently approved and marketed for the treatment of AD-associated dementia, and their utility is limited. Three of these drugs acts on central nervous system (CNS) cholinergic pathways, including donepezil, galantamine, and rivastigmine. All three drugs have anticholinesterase activity, and galantamine, which is a natural-product alkaloid, is active as an allosteric modulator at nicotinic acetylcholine receptors. Each of these drugs is now available in generic formulations and is approved for mild to severe dementia, although they are often used for patients in earlier predementia stages associated with significant progressive memory impairment based on cognitive testing results. ${ }^{42}$ Memantine is the drug most recently approved for AD in the United States and, notably, it is the first approved $\mathrm{AD}$ drug to target the N-methyl-d-aspartate (NMDA) receptor and glutaminergic pathways. Excess glutamate at excitatory synapses with associated cytotoxicity, possibly due to decreased glutamate reuptake from microglia, has recently been implicated as a pathophysiological mechanism in $\mathrm{AD}$, and glutaminergic modulation affects dendritic spine clustering in a mouse model of disease. Accordingly, riluzole, an inhibitor of glutamate release and postsynaptic glutamate receptor signaling, is in a phase II trial in mild AD patients. Chronic treatment with memantine reduces the levels of $\mathrm{A} \beta$ both in $\mathrm{AD}$ models and in aged animals, and that memantine affects the endocytosis pathway of APP, which is required for $\beta$-secretase-mediated cleavage. This leads to a reduction in $\mathrm{A} \beta$ production. Both drug memantine and donepezil have been approved in monotherapy are effected for treatment of symptoms of $\mathrm{AD}$ along with their approved indications. ${ }^{43}$ Both the drug Memantine and donepezil shows diverse and paired mechanisms of action, together they shows additional and benefits effects to the patient. Clinical study data in healthy volunteers provided initial facts that memantine and donepezil may be safely used in combination. When memantine is giving in combination of stable ChEI therapy, it shows a good safety profile in patients with $\mathrm{AD} .{ }^{43}$

\section{Non-pharmacological therapy of AD}

Non-pharmacological therapies (NPTs) can improve the quality of life (QOL) of people with Alzheimer's disease. ${ }^{44}$ There is evidence from a modest number of well-conducted randomized controlled trials that various non pharmacological approaches, including cognitive training, cognitive rehabilitation, and cognitive stimulation therapy, confer modest but significant benefits in the treatment of cognitive symptoms in people with $\mathrm{AD}$, and that there may be additive benefits in combination with cholinesterase inhibitor therapy ${ }^{45}$ Several agerelated problems with sleep and wakefulness may reflect a dampening of circadian rhythm amplitudes. Non-pharmacological manipulation of circadian rhythms by means of various external stimuli appears to be effective in improving sleep and cognitive functioning in elderly people and Alzheimer patients. ${ }^{46}$

\section{Sleep}

Disturbance in sleep seems to be a causing component of Alzheimer's disease and its pathophysiology. When sleep is improper, then it starts accumulating of amyloid- $\beta$ (A $\beta$ ), in early stage potentially it triggers the decline in memory and then convert further it to $\mathrm{AD}$, from the result of many research it has been found that Sleep patterns directly or indirectly affect Alzheimer. There are associations and plausible mechanism linking non-rapid-eye-movement (NREM) sleep disruption, $A \beta$ and AD. Disruption in NREM sleep act as novel factor linking cortical $\mathrm{A} \beta$ to impaired hippocampus dependent memory consolidation. In older age, proper sleep seems as a new treatment target, affording anticipatory and therapeutic remuneration. The bidirectional, causal interaction exists between NREM sleep and $\mathrm{A} \beta$ pathophysiology that may contribute to Alzheimer's disease risk and progression. Disruption of NREM sleep acts as a new biomarker of AD. The disruption of NREM sleep may represent a new pathway through which cortical $A \beta$ impairs hippocampus dependent memory consolidation. The disruption of NREM sleep physiology offers potential diagnostic utility in the form of non-invasive biomarker of $\mathrm{A} \beta$ pathology, $\mathrm{AD}$ risk and $\mathrm{AD}$ pathophysiological progression. Evidences implicate sleep disturbance as a consequence and cause of AD progression; one that is modifiable, offering preventative and therapeutic treatment potential. ${ }^{47}$

\section{Physical activity}

Physical and cognitive activities have been linked to indirect and direct biological factors affecting brain health. Future research will need to explore details about type, intensity, duration and combination of interventions. ${ }^{47}$ Several studies demonstrate the protective effect of Physical Activity on brain health, particularly by reducing the risk for the neurodegenerative dementia-causing AD. Other factors, including genetics, may affect the development of neurological disorders. However, in most cases, moderate PA is beneficial for both physical and mental health in older adults. Moderate-intensity aerobic exercise, resistance training, stretching, toning, and a range of motion exercises, may yield cognitive benefits in older adults. Although the exact mechanisms by which PA decreases the risk for dementia is not fully understood, PA should be encouraged, since it improves the quality of life for all older adults. The existing evidence shows that rates of dementia could be reduced, if people were physically active. There is a possibility that PA may become the most important behavioral factor in facilitating healthy mental and physical aging. Current evidence supports PA's short and long term cognitive benefits, regardless of age. ${ }^{48}$

\section{Music therapy}

Music therapy is a potential non-pharmacological treatment for the behavioral and psychological symptoms of dementia, but although some studies have found to be helpful, most are small and uncontrolled. Music therapy is a safe and effective method for treating agitation and anxiety in moderately severe and severe AD. ${ }^{49}$ Successes and failures in understanding of the pathogenesis of Alzheimer's disease 
lead to potential pitfalls in diagnosis, choice of therapeutic targets, development of drug candidates, and design of clinical trials. Several in vitro and in vivo studies are ongoing, but we need to focus on finding a proper cure for Alzheimer's disease and this forward step in the development of drugs for this disorder requires to be reconsidered. Clinical trials study is frequently provides us with new information for treatment of Alzheimer's disease, and also helps us in the analysis of patterns of pharmacological interactions as an alternative of single possible drug targets. A number of ongoing randomized controlled trials give promising results and give a way to increase collaboration between pharmaceutical companies, basic researchers, and clinical researchers who has the ability to bring us closer to developing an optimum pharmaceutical approach for the treatment of Alzheimer's disease. ${ }^{50}$

\section{Potential herbal drugs for AD}

There are common traditional Indian plants, which could be used for the treatment of Alzheimer's, dementia and other neurological disorders. Rasayana drugs of Ayurveda are rich in antioxidants and immunomodulatory agents. The strong antioxidant potential of some of these drugs has already been proven. As the majority of diseases are linked to disruption of the delicate balance between oxidants and antioxidants, the ability to scavenge free radicals or to activate oxidant defenses of cells can be thought of as their main mechanism of action. Many plants can be categorized as Rasayana plant- Ashwagandha, Brahmi, Mandukaparni, Shankapushpi, Vacha, Jatamansi and Jyotshmati. These are the herbs which are specific to brain tissues and classed as brain tonics or rejuvenators. ${ }^{51}$ Apart from above, several Rasayana drugs have been documented from our labs for beneficial effect in memory deficits and warranted for potential role in dementia and other neurological disorders and might be helpful in AD. ${ }^{52-54}$

\section{Ashwagandha}

Ashwagandha (Withania somnifera) is a shrub, part of the solanaceae family. It is considered as adaptogen- a non toxic medication that normalizes physiological functions against chronic stress by engaging the endocrinal and immune systems. Ashwagandha may help restore damaged neuronal circuits by extending its neurite outgrowth. $^{51}$

\section{Brahmi}

Brahmi (Bacopa monniera; family: Scrophularaceae) has been used for centuries as a well valued brain tonic for rejuvenating intellect, anti stress agent in anxiety, and for a means of enhancing cognitive ability. Several studies have reported that this medicinal herb acts as a mental tonic and a nervine and can be used for the treatment of mental and neurological disorders. ${ }^{51}$

\section{Gotu kola}

Gotu kola or mandookaparni (Centella asiatica) belonging to the family Apiaceae (Umbelliferae). It has been used to increase concentration, boost memory and provide alertness. Being a psychoactive medicinal plant, it is used to treat anxiety and stress. It has been used to restore youth and memory and to strengthen the nervous system. It has been used to improve memory. ${ }^{51}$

\section{Chandan}

Chandan (Santalum album) belonging to family Santalaceae. The potential of this plant is to increase memory and cognitive function (as described in siddha). Licorice has significant action on memory enhancing activity and significantly improves learning and memory in scopolamine- induced dementia. ${ }^{51}$

\section{Bhilawa}

Bhilawa (Semencarpus anacardium) belonging to the family Anacardiaceae. It acts as a brain tonic and is a potent antioxidant agent. ${ }^{51}$

\section{Haldi}

Haldi (Curcuma longa) is used for the treatment of most of chronic illnesses, including neurodegenerative, cardiovascular, pulmonary, metabolic, autoimmune and neoplastic diseases. ${ }^{51}$ Curcumin is a therapeutic potential herbal drug in $\mathrm{AD}$. Curcumin (the major chemical constituent of Haldi or Turmeric) when administered in a mouse model of $\mathrm{AD}$, it decreased in serum $\mathrm{A} \beta$ level as well as minimizes burden of $A \beta$ in the brain, and this effect is seen mainly in the neocortex and hippocampus of the AD mouse model. ${ }^{55}$ Curcumin crosses the blood-brain barrier and it inhibits the formation of $A \beta$ plaques and it dissolves of preformed $A \beta$ fibrils and it inhibits their extension. Curcumin treatment is capable of recovering the deformed neuritic morphology present near plaques and gives better therapeutic effect in $\mathrm{AD}$. It is more effective in inhibiting the $\mathrm{A} \beta$ plaques formation than naproxen or ibuprofen. ${ }^{56-58}$ Production and growth of amyloid precursor protein (APP) is another pathway in disruption of $\mathrm{AD} .^{59}$ Curcumin used to minimize the production of $A \beta$ with a similarly minimise in presenilin1 gene expression, which suggests its role in $\gamma$-secretase modulation. ${ }^{60}$ Moreover, it is also possible that the APP production is decreased due to curcumin's effect on APP maturation. ${ }^{61}$

It is interesting to know that curcumin and some metabolites of it have shown therapeutic potential for AD.$^{62}$ Most frequently used metabolites of curcumin are tetrahydrocurcumin, hexahydrocurcumin, and octahydrocurcumin these are reductive in nature. ${ }^{62,63}$ On the other hand, less studied metabolic products of bisdemethoxy curcumin and demethoxy curcumin rather than curcumin. When tetrahydrocurcumin and curcumin are compared then only curcumin was effective in reducing plaque burden. Under in vivo conditions, tetrahydrocurcumin was shown to reduce the neuroinflammation but ineffective in reducing plaques and insoluble $A \beta$. Other metabolite of the Curcumin is Ferulic acid, which is known to possess antioxidant effect. ${ }^{62}$ Tetrahydrocurcumin in recent studies showed protection, predominantly against $A \beta$-oligomer-induced toxicity, which was its antioxidant activity ${ }^{64-66}$ and anti-inflammatory activities. ${ }^{67,68}$ Ferulic acid also an essential metabolite in reducing the production of $\mathrm{A} \beta$ plaques and extension from amyloid beta-peptide in a dosedependent manner. ${ }^{69}$ Under the in vivo conditions in a transgenic $\mathrm{AD}$ mouse model ( $\operatorname{Tg} 2576)$ ferulic acid did not show a therapeutically significant effect on the amyloid profile because ferulic acid is not able to penetrate the brain effectively. ${ }^{70}$

\section{Risk management of AD}

Recent studies showed that improved physical and cognitive functioning might contribute to decline of the dementia prevalence. Currently, no cure exists in $\mathrm{AD}$, highlighting the need to reduce the incidence of this devastating disease by identifying modifiable risk factors. Several studies have demonstrated that sedentary lifestyles, hypertension, and lifestyle-associated conditions such as obesity and Type 2 diabetes mellitus contribute to increased risk of $\mathrm{AD} .^{71}$ 
Biochemical exposure (hyperhomocysteine) and psychological condition (depression) significantly increase risk of developing AD. Studies indicated complex roles of pre-existing disease (frailty, carotid atherosclerosis, hypertension, low DBP, DM-2 (Asian population) increasing risk, whereas a history of arthritis, heart disease, metabolic syndrome and cancer decreasing risk) and lifestyle (low education, high BMI in mid-life and low BMI increasing the risk whereas cognitive activity, current smoking (cigarette smoke consists of more than 4500 toxic chemicals that combine to form free radicals, which lead to oxidative stress-associated neurological disorders). ${ }^{72}$ Lightto-moderate drinking, stress, high BMI in late-life decreasing the risk) in influencing $\mathrm{AD}$ risk. ${ }^{73}$ The etiology of $\mathrm{AD}$ is still not clear; however, the role of the environment as a supposed risk factor has gained significance. Moreover, the evidences supported that preand post-natal exposures to environmental factors predispose to the onset of neurodegenerative diseases in later life. Neurotoxic metals such as lead, mercury, aluminum, cadmium and arsenic, as well as some pesticides and metal-based nanoparticles have been involved in $\mathrm{AD}$ due to their ability to increase beta-amyloid $(\mathrm{A} \beta)$ peptide and the phosphorylation of Tau protein (P-tau), causing senile/amyloid plaques and neurofibrillary tangles (NFTs) characteristic of AD. ${ }^{74}$ There are various genetic risk factors for the onset of Alzheimer's disease and their role in AD pathogenesis. More recent advances in understanding of the human genome, technological advances in methods to analyze millions of polymorphisms in thousands of subjects have revealed new genes associated with AD risk, including ABCA7, BIN1, CASS4, CD33, CD2AP, CELF1, CLU, CR1, DSG2, EPHA1, FERMT2, HLA-DRB5-DBR1, INPP5D, MS4A, MEF2C, NME8, PICALM, PTK2B, SLC24H4-RIN3, SORL1 and ZCWPW1. Emerging technologies to analyze the entire genome in large data sets have also revealed coding variants that increase AD risk: PLD3 and TREM2. ${ }^{75}$ Who are carriers of the $\varepsilon 4$ allele of apolipoprotein $\mathrm{E}$ in healthy adult is the prevailing genetic risk factor for late-onset Alzheimer's disease, the adults who are on self-referential processing and emotional processing to the same degree and benefited as non carriers of this gene. The magnitude of memory effect is not affected by $\varepsilon 4$ status. It shows beneficial effect and emotional enhancement in older adult $\varepsilon 4$ non-carriers rather than older adult $\varepsilon 4$ carriers. Therefore, deficits in emotional memory may be an early cognitive marker of abnormal decline in cognitively healthy older adult's $\varepsilon 4$ carriers following self-reference. There is no evidence suggesting a significant association of AD with occupational exposures. Therefore, effective interventions in diet, medications, biochemical exposures, psychological condition, pre-existing disease, and lifestyle may lower new incidence of $\mathrm{AD} .^{73}$

\section{Concluding remarks}

As reviewed in this paper, the increased global burden of $\mathrm{AD}$ leading cause of death and disability worldwide. Apart from available therapeutic targets, biomarkers and pharmaco-therapies available, the search of complete management of AD is enduring. However, combination of pharmacotherapy and non-pharmacological treatments approach are praiseworthy. Herbal drugs and secondary metabolites are also playing beneficial role in the management of $\mathrm{AD}$. Therefore, robust pre-clinical studies and clinical trials for new drug target and potential drug candidate are warranted for complete management of $\mathrm{AD}$.

\section{Acknowledgements}

None.

\section{Conflict of interest}

The author declares that there is no conflict of interest.

\section{References}

1. Kumar A, Singh A, Ekavali. A review on Alzheimer's disease pathophysiology and its management: an update. Pharmacol Rep. 2015;67(2):195-203.

2. Vinters HV. Emerging Concepts in Alzheimer's Disease. Annu Rev Pathol. 2015;10:291-319.

3. Bird TD, Miller BL. Alzheimer's disease and primary dementias. In: Fauci AS, Braunwald E, editors. Harrison's Principles of Internal Medicine. 17th ed. New York: McGraw-Hill; 2008:2393-2406.

4. Ahmed T, Gilani AH. Therapeutic potential of turmeric in Alzheimer's disease: curcumin or curcuminoids? Phytother Res. 2014;8(4):517-525.

5. Burns A, Iliffe S. Alzheimer's disease. BMJ. 2009;338:b158.

6. Wimo A, Jonsson L, Bond J, et al. The worldwide economic impact of dementia 2010. Alzheimers Dement. 2013;9(1):1-11.

7. Mishra S, Palanivelu K. The effect of curcumin (turmeric) on Alzheimer's disease: An overview. Ann Indian Acad Neurol. 2008;11(1):13-19.

8. Liu Z, Zhang A, Sun H, et al. Two decades of new drug discovery and development for Alzheimer's disease. RSC Advances. 2017;7(10):60466058 .

9. Mohamed T, Shakeri A, Rao PPN. Amyloid cascade in Alzheimer's disease: Recent advances in medicinal chemistry. Eur J Med Chem. 2016;113:258-272.

10. Weekley CM, He C. Developing drugs targeting transition metal homeostasis. Curr Opin Chem Biol. 2017;37:26-32.

11. Prakash A, Dhaliwal GK, Kumar P, et al. Brain biometals and Alzheimer's disease - boon or bane? Int J Neurosci. 2017;127(2):99-108.

12. Sultzer DL, Marder SR. Older Brains are Different: Brain-Behavior Studies and Their Clinical Utility. Am J Geriatr Psychiatry. 2017;25(1):11-12.

13. Chase TN, Farlow MR, Clarence-Smith K. Donepezil Plus Solifenacin (CPC-201) Treatment for Alzheimer's Disease. Neurotherapeutics. 2017;14(2):405-416.

14. Sarazin M, de Souza LC, Lehéricy S, et al. Clinical and Research Diagnostic Criteria for Alzheimer's Disease. Neuroimaging Clin N Am. 2012;22(1):23-32.

15. Yaari R, Fleisher AS, Tariot PN. Updates to Diagnostic Guidelines for Alzheimer's Disease. Prim Care Companion CNS Disord. 2011;13(5).

16. Barage SH, Sonawane KD. Amyloid cascade hypothesis: Pathogenesis and therapeutic strategies in Alzheimer's disease. Neuropeptides. 2015;52:1-18.

17. Lovheim H, Gilthorpe J, Adolfsson R, et al. Reactivated herpes simplex infection increases the risk of Alzheimer's disease. Alzheimers Dement. 2015;11(6):593-599.

18. Khachaturian ZS. Diagnosis of Alzheimer's Disease. Arch Neurol. 1985;42(11):1097-1105.

19. Delacourte A. Diagnosis of Alzheimer's disease. Ann Biol Clin (Paris). 1998;56(2):133-142.

20. Dubois B, Feldman HH, Jacova C, et al. Research criteria for the diagnosis of Alzheimer's disease: revising the NINCDS-ADRDA criteria. Lancet Neurol. 2007;6(8):734-746.

21. Sharma S, Lipincott W. Biomarkers in Alzheimer's Disease-Recent Update. Curr Alzheimer Res. 2017. 
22. Blennow K. Biomarkers in Alzheimer's disease drug development. Nat Med. 2010;16(11):1218-1222.

23. Heinrich M, Lee Teoh H. Galanthamine from snowdrop-the development of a modern drug against Alzheimer's disease from local Caucasian knowledge. J Ethnopharmacol. 2004;92(2-3):147-162.

24. Shu YZ. Recent natural products based drug development: a pharmaceutical industry perspective. J Nat Prod. 1998;61(8):1053-1071.

25. Aso E, Ferrer I. Cannabinoids for treatment of Alzheimer's disease: moving toward the clinic. Front Pharmacol. 2014;5:37.

26. Dodel R, Hampel H, Depboylu C, et al. Human antibodies against amyloid beta peptide: a potential treatment for Alzheimer's disease. Ann Neurol. 2002;52(2):253-256.

27. Guzior N, Wieckowska A, Panek D, et al. Recent development of multifunctional agents as potential drug candidates for the treatment of Alzheimer's disease. Curr Med Chem. 2015;22(3):373-404.

28. Hayne DJ, Lim S, Donnelly PS. Metal complexes designed to bind to amyloid-[small beta] for the diagnosis and treatment of Alzheimer's disease. Chem Soc Rev. 2014;43(19):6701-6715.

29. Wang J, Bi W, Cheng A, et al. Targeting multiple pathogenic mechanisms with polyphenols for the treatment of Alzheimer's disease-experimental approach and therapeutic implications. Front Aging Neurosci. 2014;6:42.

30. Forlenza OV, De-Paula VJR, Diniz BSO. Neuroprotective Effects of Lithium: Implications for the Treatment of Alzheimer's Disease and Related Neurodegenerative Disorders. ACS Chem Neurosci. 2014;5(6):443-450.

31. Woods LT, Ajit D, Camden JM, et al. Purinergic receptors as potential therapeutic targets in Alzheimer's disease. Neuropharmacology. 2016;104:169-179.

32. Kumar A, Nisha CM, Silakari C, et al. Current and novel therapeutic molecules and targets in Alzheimer's disease. J Formos Med Assoc. 2016;115(1):3-10.

33. Guerram M, Zhang LY, Jiang ZZ. G-protein coupled receptors as therapeutic targets for neurodegenerative and cerebrovascular diseases. Neurochem Int. 2016;101:1-14.

34. Adeniji AO, Adams PW, Mody VV. Amyloid $\beta$ Hypothesis in the Development of Therapeutic Agents for Alzheimer's Disease. In: Adejare A, editor. Drug Discovery Approaches for the Treatment of Neurodegenerative Disorders. Philadelphia: Academic Press; 2017:109143.

35. Wang J, Chen GJ. Mitochondria as a therapeutic target in Alzheimer's disease. Genes \& Diseases. 2016;3(3):220-227.

36. Hansen RA, Gartlehner G, Kaufer D, et al. Drug Class Review of Alzheimer's Drugs. Final Report. 2006

37. Scarpini E, Scheltens P, Feldman H. Treatment of Alzheimer's disease: current status and new perspectives. Lancet Neurol. 2003;2(9):539-547.

38. Sramek JJ, Cutler NR. Recent developments in the drug treatment of Alzheimer's disease. Drugs Aging. 1999;14(5):359-373.

39. Geldenhuys WJ, Darvesh AS. Pharmacotherapy of Alzheimer's disease: current and future trends. Expert Rev Neurother. 2015;15(1):3-5.

40. Adwan L, Zawia NH. Epigenetics: a novel therapeutic approach for the treatment of Alzheimer's disease. Pharmacol Ther. 2013;139(1):41-50.

41. Freiherr J, Hallschmid M, Frey WH, et al. Intranasal insulin as a treatmen for Alzheimer's disease: a review of basic research and clinical evidence. CNS Drugs. 2013;27(7):505-514

42. Graham WV, Bonito-Oliva A, Sakmar TP. Update on Alzheimer's Disease Therapy and Prevention Strategies. Annu Rev Med. 2017;68:413-430.
43. Ito K, Tatebe T, Suzuki K, et al. Memantine reduces the production of amyloid-beta peptides through modulation of amyloid precursor protein trafficking. Eur J Pharmacol. 2017;798:16-25.

44. Olazaran J, Reisberg B, Clare L, et al. Nonpharmacological therapies in Alzheimer's disease: a systematic review of efficacy. Dement Geriatr Cogn Disord. 2010;30(2):161-178.

45. Ballard C, Khan Z, Clack H, et al. Nonpharmacological treatment of Alzheimer disease. Can J Psychiatry. 2011;56(10):589-595.

46. van Someren EJW, Mirmiran M, Swaab DF. Non-pharmacological treatment of sleep and wake disturbances in aging and Alzheimer's disease: Chronobiological perspectives. Behav Brain Res. 1993;57(2):235-253.

47. Lautenschlager NT, Anstey KJ, Kurz AF. Non-pharmacological strategies to delay cognitive decline. Maturitas. 2014;79(2):170-173.

48. Gallaway PJ, Miyake H, Buchowski MS, et al. Physical Activity: A Viable Way to Reduce the Risks of Mild Cognitive Impairment Alzheimer's Disease, and Vascular Dementia in Older Adults. Brain Sci. 2017;7(2):E22.

49. Svansdottir HB, Snaedal J. Music therapy in moderate and severe dementia of Alzheimer's type: a case-control study. Int Psychogeriatr. 2006;18(4):613-621.

50. Mangialasche F, Solomon A, Winblad B, et al. Alzheimer's disease: clinical trials and drug development. Lancet Neurol. 2010;9(7):702-716.

51. Farooqui T, Farooqui AA. Neuroprotective Effects of Phytochemicals in Neurological Disorders. Wiley Blackwell: USA; 2017.

52. Thakur AK, Chatterjee SS, Kumar V. Beneficial effects of Brassica juncea on cognitive functions in rats. Pharm Biol. 2013;51(10):1304-1310.

53. Thakur AK, Rai G, Chatterjee SS, et al. Beneficial effects of an Andrographis paniculata extract and andrographolide on cognitive functions in streptozotocin-induced diabetic rats. Pharm Biol. 2016;54(9):1528-1538

54. Thakur AK, Raj P. Pharmacological Perspective of Glycyrrhiza glabra Linn.: a Mini-Review. J Anal Pharm Res. 2017;(5):00156.

55. Wang SL, Li Y, Wen Y, et al. Curcumin, a potential inhibitor of upregulation of TNF-alpha and IL-6 induced by palmitate in 3T3-L1 adipocytes through NF-kappaB and JNK pathway. Biomed Environ Sci. 2009;22(1):32-39.

56. Garcia-Alloza M, Borrelli LA, Rozkalne A, et al. Curcumin labels amyloid pathology in vivo, disrupts existing plaques, and partially restores distorted neurites in an Alzheimer mouse model. J Neurochem. 2007;102(4):1095-1104.

57. Ono K, Hasegawa $\mathrm{K}$, Naiki $\mathrm{H}$, et al. Curcumin has potent antiamyloidogenic effects for Alzheimer's beta-amyloid fibrils in vitro. $J$ Neurosci Res. 2004;75(6):742-750.

58. Yang F, Lim GP, Begum AN, et al. Curcumin inhibits formation of amyloid beta oligomers and fibrils, binds plaques, and reduces amyloid in vivo. J Biol Chem. 2005;280(7):5892-5901.

59. Suh YH, Checler F. Amyloid precursor protein, presenilins, and alphasynuclein: molecular pathogenesis and pharmacological applications in Alzheimer's disease. Pharmacol Rev. 2002;54(3):469-525.

60. Xiong HY, Barash Y, Frey BJ. Bayesian prediction of tissue-regulated splicing using RNA sequence and cellular context. Bioinformatics. 2011;27(18):2554-2562.

61. Zhang C, Browne A, Child D, et al. Curcumin decreases amyloid-beta peptide levels by attenuating the maturation of amyloid-beta precursor protein. J Biol Chem. 2010;285(37):28472-28480.

62. Begum AN, Jones MR, Lim GP, et al. Curcumin structure-function, 
bioavailability, and efficacy in models of neuroinflammation and Alzheimer's disease. J Pharmacol Exp Ther. 2008;326(1):196-208.

63. Hoehle SI, Pfeiffer E, Solyom AM, et al. Metabolism of curcuminoids in tissue slices and subcellular fractions from rat liver. J Agric Food Chem. 2006;54(3):756-764.

64. Graf E. Antioxidant potential of ferulic acid. Free Radic Biol Med. 1992;13(4):435-448.

65. Mishra S, Mishra M, Seth P, et al. Tetrahydrocurcumin confers protection against amyloid beta-induced toxicity. Neuroreport. 2011;22(1):23-27.

66. Scott BC, Butler J, Halliwell B, et al. Evaluation of the antioxidant actions of ferulic acid and catechins. Free Radic Res Commun. 1993;19(4):241253.

67. Fernandez MA, Saenz MT, Garcia MD. Anti-inflammatory activity in rats and mice of phenolic acids isolated from Scrophularia frutescens. $J$ Pharm Pharmacol. 1998;50(10):1183-1186.

68. Ozaki Y. Antiinflammatory effect of tetramethylpyrazine and ferulic acid. Chem Pharm Bull (Tokyo). 1992;40(4):954-956.

69. Ono K, Hirohata M, Yamada M. Ferulic acid destabilizes preformed $\beta$ amyloid fibrils in vitro. Biochem Biophys Res Commun. 2005;336(2):444 449.
70. Hamaguchi T, Ono K, Murase A, et al. Phenolic compounds prevent Alzheimer's pathology through different effects on the amyloid-beta aggregation pathway. Am J Pathol. 2009;175(6):2557-2565.

71. Jayaraman A, Pike CJ. Alzheimer's disease and type 2 diabetes: multiple mechanisms contribute to interactions. Curr Diab Rep. 2014;14(4):476.

72. G SB, Choi S, Krishnan JKR. Cigarette smoke and related risk factors in neurological disorders: An update. Biomed Pharmacother. 2017;85:7986

73. Xu W, Tan L, Wang HF, et al. Meta-analysis of modifiable risk factors for Alzheimer's disease. J Neurol Neurosurg Psychiatry. 2015;86(12):12991306.

74. Chin-Chan M, Navarro-Yepes J, Quintanilla-Vega B. Environmental pollutants as risk factors for neurodegenerative disorders: Alzheimer and Parkinson diseases. Frontiers in Cellular Neuroscience. 2015;9:124.

75. Karch CM, Goate AM. Alzheimer's disease risk genes and mechanisms of disease pathogenesis. Biol Psychiatry. 2014;77(1):43-51.

76. Grilli MD, Woolverton CB, Crawford Ms, et al. Self-reference and emotional memory effects in older adults at increased genetic risk of Alzheimer's disease. Aging Neuropsychol Cogn. 2017;25(2):186-199. 